\title{
Editorial
}

\section{Educación intercultural: memorias, víctimas del conflicto y experiencias educativas}

\author{
Jenny Paola Ortiz Fonseca
}

$\mathrm{L}$ a educación intercultural es una perspectiva ética, política y epistémica que nos permite problematizar y combatir las condiciones históricas de desigualdad de los sectores subalternos. Consideramos que desde este punto de vista se han problematizado y complejizado permanentemente algunos núcleos de análisis: el problema de la cultura, las relaciones de poder, el lugar que ocupan los sujetos y las colectividades en la jerarquía de la diferencia, el colonialismo/modernidad y el cuestionamiento a la matriz colonial que ha configurado un proyecto de sociedad racista, excluyente, sexista y clasista.

Desde estos núcleos de análisis es necesario cuestionar los lugares de representación que se han construido alrededor de los grupos subalternos, sus memorias, sus identidades, sus diferencias y sus intersecciones en el escenario educativo. Justamente, estos aspectos son abordados en este número de la revista Controversia, donde nos hacemos las siguientes preguntas: ipor qué es importante hablar de la relación entre las memorias subalternas y las víctimas del conflicto armado en el actual proceso de transición política que vive el país?, ¿qué tipos de procesos pedagógicos interculturales se pueden desarrollar frente al reconocimiento de los derechos de los grupos étnicos?

Estas cuestiones emergen de dos ejes reflexivos fundamentales:

- La articulación entre memoria, historia y víctimas del conflicto armado, en la perspectiva del derecho a la verdad, al perdón y a la reparación, en el actual proceso de transición política y de construcción de paz territorial. 
- La reflexión crítica sobre algunos procesos educativos que problematizan y complejizan el quehacer pedagógico, el currículo, la formación docente y los dispositivos pedagógicos en ejercicios del reconocimiento a las identidades, diferencias y narrativas étnico-raciales.

Los tres primeros artículos estarán situados desde el primer eje reflexivo, y nos presentarán, a su vez, tres aspectos:

a) El debate por las memorias y la historia de los sectores subalternos/ subalternizados en la perspectiva del derecho a la verdad y en la necesidad que tenemos en Colombia, como sociedad, de explicar y comprender en sus múltiples dimensiones los impactos del conflicto armado en el país.

b) La reflexión sobre las víctimas del conflicto armado, en clave de generar mecanismos de ruptura con los estereotipos y marcaciones identitarias que reproducen miradas revictimizantes, así como la importancia que tiene para este propósito la relación entre el perdón, la justicia y la reparación integral que nos permita reconstruirnos como sociedad colombiana.

c) El lugar que ocuparía la escuela en la reconstrucción del tejido social, donde se pueden visibilizar, tramitar y generar espacios que faciliten la reparación integral de niños, niñas y jóvenes víctimas del conflicto armado.

Mauricio Archila, en su texto "Memoria, verdad e historia oral”, centra la atención en el derecho de las víctimas del conflicto armado a la verdad y a los usos públicos de la historia en la coyuntura actual. Toma como punto de partida tres interrogantes provocadores: de qué memoria hablamos y cómo se se relaciona con la disciplina histórica, cuál es la verdad a la que apuntan los trabajos de la memoria, y qué papel juega la historia oral en estos procesos.

En la actual transición para la construcción de la paz territorial se hace fundamental situarnos desde el lugar del derecho de las víctimas del conflicto armado colombiano a la verdad, la justicia, la no repetición y 
una vida digna. Esto significa que, cuando se plantea la discusión sobre los trabajos por las memorias y la historia como disciplina, se hace desde los grupos subalternos que ocupan múltiples y simultáneos espacios de exclusión, explotación y opresión. La memoria y la historia son prácticas de construcción del pasado y se relacionan entre sí. La primera se ocupará del recuerdo como un acto subjetivo que le da sentido a la experiencia individual y colectiva, mientras que la segunda buscará explicar y comprender los procesos y fenómenos sociales como parte del campo de la disciplina histórica. Desde ambas perspectivas, se puede resaltar que las memorias subalternas luchan por la verdad, se enfrentan por el pasado y sus narrativas, y permiten reafirmar las identidades colectivas.

Aunado a esta reflexión, se sitúa el artículo de Laura Henao, titulado "Análisis sociológico del perdón: discursos dominantes y alternativos", donde se plantea que en el contexto actual de transición política es fundamental problematizar las narrativas del perdón y su potencia política en el efecto de reparación a víctimas del conflicto armado.

Henao está permanentemente en una disertación sociológica respecto a los distintos abordajes del perdón (jurídico, sociológico y político) y sus relaciones con las diversas experiencias transicionales en el mundo. En sus análisis identifica que las narrativas que se han construido desde la prensa y los medios de comunicación difunden unos relatos que contrastan con las miradas y las narraciones de quienes han sufrido el conflicto armado. Tanto la construcción de la verdad como el perdón se convierten en categorías que están en pugna por comprender y tramitar los hechos traumáticos del pasado y son escenarios que permiten la reparación integral de las víctimas del conflicto desde la no repetición y la construcción de narrativas subalternas que rompan con las marcaciones y victimizaciones de odio que han marcado el curso de nuestro país.

En el artículo de Lorena Torres, “Agenciamiento de la memoria social en la escuela colombiana como mecanismo reparador”, también se hace un abordaje al perdón y la reparación integral de niños, niñas y jóvenes 
víctimas del conflicto armado en Colombia desde el papel de la escuela. La autora problematiza la relación entre agenciamiento, escuela, memoria y reparación en el contexto nacional de justicia transicional, el lugar que ocupa la escuela para visibilizar, tramitar y gestionar el reconocimiento de las narrativas de las víctimas del conflicto armado y la potencia política que tiene para generar escenarios de transformación. Este propósito se desarrolla desde dos elementos reflexivos: por un lado, el primero, la reparación como responsabilidad social que nos compromete a todos como sociedad en la construcción de escenarios de paz y su relación con la escuela en la perspectiva de formar en y para la paz y la convivencia, y el segundo, por otro lado, se sitúa en el agenciamiento de la memoria como una apuesta por la reparación y los lugares que ocuparía la memoria social en la escuela para construir diversas narrativas del conflicto desde las voces de las víctimas.

La relación entre agenciamiento y memoria es un mecanismo que permite resignificar y complejizar las narrativas que se han elaborado sobre la categoría de víctima. En este sentido, se plantea que los ejercicios de memoria permiten problematizar las narrativas que se han construido sobre una sola historia, han revictimizado a las poblaciones que padecieron la guerra y, sobre todo, complejizan el lugar que ocuparía la escuela para crear escenarios reflexivos sobre la reparación integral a las víctimas del conflicto armado. Es fundamental comprender y afianzar las propuestas escolares desde las pedagogías de la memoria, en cuanto invitan a generar espacios de formación desde la historia reciente de una sociedad que enfrentó el conflicto armado e inició procesos transicionales en búsqueda de paz. Las pedagogías de la memoria hacen evidente el papel activo de los sujetos en su proceso identitario tanto en la transmisión como en la apropiación de las memorias sociales y los diferentes lugares que le otorgamos a la educación.

Los siguientes tres artículos sitúan sus reflexiones desde propuestas educativas que se desarrollan en el Caribe colombiano, el Cauca, y en Oaxaca, México. Los autores nos invitan a pensar el lugar que ocupa la 
diferencia étnica y cultural en el escenario escolar desde tres dimensiones centrales:

a) El posicionamiento que tienen los docentes en tres sentidos: el primero, está situado desde el reconocimiento de la propia experiencia histórica de los docentes en claves étnico-racial y de género; el segundo, hace referencia al proceso de formación y quehacer pedagógico, donde se reconoce o invisibiliza la diferencia étnica-racial en el aula de clases; y, finalmente, en el tercero se complejiza la relación existente entre la escuela como una institución normalizadora y como un espacio de fortalecimiento étnico y cultural.

b) Las fronteras y los límites que afrontan la construcción de currículos y las propuestas educativas desde los pueblos indígenas y afrodescendientes, que permanentemente se mueven en la ambivalencia entre el conocimiento propio y los lineamientos establecidos por el Ministerio de Educación Nacional (MEN).

c) La propuesta para combatir la desigualdad epistémica del conocimiento entre uno, pretendidamente universal, y los “otros” marginados o subalternizados. Estas propuestas se sitúan en la tensión constante entre el multiculturalismo y la interculturalidad en el escenario escolar.

Como resultado del proceso de formación y acompañamiento realizado por el Cinep en la región Caribe (Sierra Nevada, sur de La Guajira y Cartagena) y de una investigación de corte etnográfica, Leidy Perneth presenta su artículo "Violencias epistémicas y acciones pedagógicas de resistencia en el Estado multicultural”. En él centra su atención en las tensiones entre el enfoque intercultural y de género presentes en la formación docente y el quehacer pedagógico. La autora sitúa cuatro tensiones entendidas como experiencias conflictivas que han interpelado a los docentes durante su quehacer pedagógico: a) las fronteras entre etnoeducación, educación propia y las políticas educativas, donde han sido centrales los conflictos que tienen los y las docentes al enseñar 
conocimientos hegemónicos y conocimientos subalternos; b) los retos que tienen la etnoeducación y la interculturalidad frente al sistema evaluativo; c) la institucionalización de la etnoeducación como parte de las políticas de Estado, que gestiona la diferencia étnica y cultural desde las miradas multiculturales y las políticas neoliberales; y d) la discusión de un currículo oficial y un currículo subalterno como los puntos de fuga y de borde que realizan los docentes en su quehacer pedagógico.

Siguiendo esta línea, se encuentra el artículo "Notas acerca de la construcción curricular en la educación indígena”, de Santiago Gutiérrez, donde el autor presenta diversas reflexiones provocadoras a partir del trabajo realizado con organizaciones indígenas en el Cauca durante 2015 y 2016. Estas notas parten del supuesto de que la historia de la educación de los pueblos indígenas está cruzada por una relación constante entre dominación y resistencia, en la que se pueden identificar al menos tres grandes momentos: a) el proyecto de evangelización (siglos XVII y XVIII) que se movilizaría sobre los principios civilizatorios y religiosos de la colonización; b) los inicios del siglo XIX con la educación para pueblos indígenas desde la perspectiva del integracionismo escolar mediante la institucionalización de las misiones religiosas; y c) el periodo de los derechos étnicos que comienza a mediados del siglo XX y que se rectifica con la Constitución del 1991 y las normativas subsecuentes, donde se dan dos situaciones simultáneas: el reconocimiento de la diversidad étnica y la regulación de la diferencia por parte del Estado-nación.

Desde este supuesto, las notas propuestas por el autor problematizan tres tensiones que emergen en la construcción de escuelas propias, currículos, la relación entre los modelos educativos propios y los lineamientos del MEN que ponen en discusión diversas complejidades que afrontan las instituciones educativas en el Cauca. La primera de dichas tensiones se relaciona con la ambivalencia entre los procedimientos y procesos de las políticas del Estado, los planes educativos y la evaluación, que ponen en conflicto la construcción de currículos de educación 
propia frente a la normalización de las identidades culturales expresadas en los lineamientos del MEN. La segunda tensión está vinculada con las modalidades de contratación (docentes provisionales, de propiedad y contratados por las organizaciones), que están permanentemente en disputa por la construcción curricular, las exigencias de las organizaciones y el sentido de la educación en comunidades indígenas. Y la tercera se sitúa en los límites que debe ocupar la escuela entre la educación formal y no formal, es decir, los bordes en los que se mueven permanentemente las instituciones educativas entre las propuestas de educación propia y los lineamientos de educación del MEN.

"Volver a tu tierra: dispositivo pedagógico intra y entrecultural en la formación docente inicial en Oaxaca, México” es resultado de la reflexión sobre el proceso de formación docente en la Escuela Normal Rural Vanguardia Tamazulápam del Progreso en Oaxaca, México. El autor, de forma recurrente, plantea la necesidad de situar a los docentes en formación como un producto-productor con una trayectoria histórica que interpela su lugar de agencia en el proceso formativo. En este sentido, se trata de hacer un ejercicio de la propia construcción de la experiencia docente en relación con sus construcciones de identidad. Lo anterior permite situar la práctica pedagógica como un complejo proceso de reconocimiento desde la experiencia histórica de los docentes y la relación que tendría con el fortalecimiento cultural en escenarios educativos indígenas. Estas reflexiones de los docentes en formación permiten potenciar su actuar pedagógico reconociendo las múltiples identidades y diferencias que están presentes en los territorios indígenas de Oaxaca.

El autor propone que Volver a la tierra es un dispositivo pedagógico que les permite a los docentes en formación regresar a sus lugares de origen para realizar las prácticas pedagógicas, lo que les ayuda a problematizar los escenarios escolares más allá de los planes de estudios, es decir, situar el proceso educativo como un escenario de formación abierto e intersubjetivo entre estudiantes y docentes en formación. De allí que el autorreconocimiento se convierta en un lugar importante en 
los asuntos de orden epistemológico y ontológico de las comunidades indígenas desde su propia experiencia histórica, el autorreconocimiento de su identidad y la necesidad de pensar otras historias, geografías, lenguajes, entre otros, que estén vinculados o creen rupturas con los procesos de aprendizaje que se sitúan desde el conocimiento occidental y eurocéntrico.

Finalmente, los diversos artículos que hacen parte de este número de Controversia nos insinúan tres retos que debemos continuar pensando y reflexionando:

- Un primer reto para afrontar como sociedad colombiana se refiere a los trabajos por la memoria y la historia que serán centrales para explicar y comprender la verdad sobre el conflicto armado, sobre las voces de las víctimas y como ejercicio esclarecedor que nos permita reconstruirnos como sociedad colombiana.

- El segundo reto está centrado en la necesidad de pensar las intersecciones que existen entre víctimas, grupos étnicos, mujeres y educación como escenarios que permiten complejizar las relaciones, las resistencias y las propuestas que se generan desde estos lugares de encuentro y desencuentro.

- El tercer reto es el lugar que tiene la educación intercultural para problematizar y hacer visibles los distintos lugares de opresión que ocupan los sectores subalternos, así como el reconocimiento de propuestas que desde los bordes invitan a la acción transformadora desde la escuela para romper con la matriz colonial y hegemónica.

En resumen, esperamos que los artículos de las siguientes páginas aporten a la reflexión sobre los procesos de educación intercultural y contribuyan a problematizar las condiciones de desigualdad que han ocupado históricamente los sectores subalternos. 